CORRIGENDUM

doi:10.1038/nature 10222

\title{
Chromatin regulation by Brg1 underlies heart muscle development and disease
}

Calvin T. Hang, Jin Yang, Pei Han, Hsiu-Ling Cheng,

Ching Shang, Euan Ashley, Bin Zhou \& Ching-Pin Chang

Nature 466, 62-67 (2010)

In this Letter, a panel in Fig. $4 \mathrm{f}$ (thymus chromatin immunoprecipitation (ChIP) input lane) was inadvertently duplicated in a panel in Fig. $4 \mathrm{~m}$ (hearts with transverse aortic constriction (TAC) ChIP input lane). The corrected input lane for Fig. 4m, shown below, was from Supplementary Fig. 11f because the ChIPs for histone deacetylases 2 and 9 (HDAC2 and HDAC9) were conducted in the same experiment. Our practice is to establish an optimal polymerase chain reaction (PCR) condition for each sample so that all inputs attain a similar level of amplification regardless of tissue source, allowing normalization and cross-comparison among different samples. Because normalization involved only sonicated genomic DNA prior to immunoprecipitation, the bands from the input gels were nearly identical in appearance for both heart and thymus, and this unfortunately caused us to misplace the two input figures during manuscript preparation.

Also, in Fig. $5 c$ the $y$-axis is mislabelled and should be ' $\beta-M H C / \alpha$ $M H C$ mRNA ratio (y)' and the mathematical formula should be $y=150 e^{-e^{160-110 x}}$ (the corrected Fig. $5 \mathrm{c}$ is shown below). These changes do not affect the overall conclusion of the manuscript. Nor do they affect our specific conclusions that the chromatin-remodelling protein Brgl does not bind to myosin heavy chain (MHC)- $\alpha$ or MHC- $\beta$ promoters in thymus, whereas HDAC2 and HDAC9 bind to the same promoters in stressed adult hearts, and the mathematical model describing Brg1 and MHC expression.
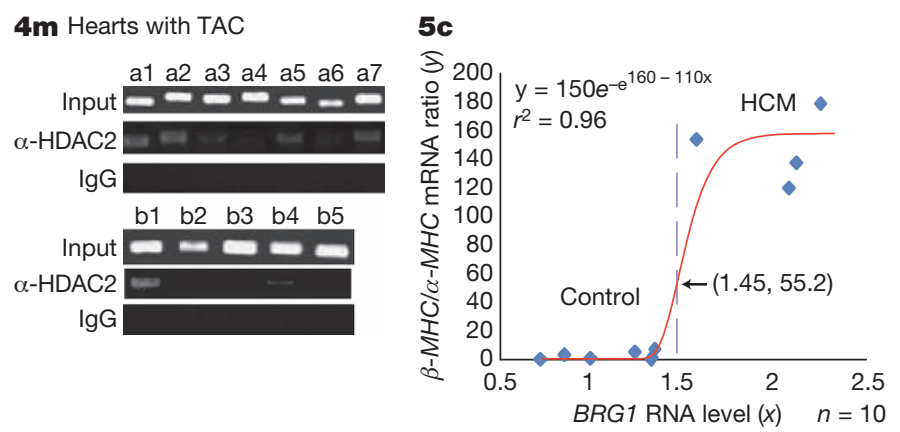